\title{
Ultrasonido y accesos venosos periféricos difíciles: de las barreras al éxito, un reporte de casos
}

\section{Ultrasound and difficult peripheral venous access: from barriers to success, a case report}

\author{
John Camilo García Uribe ${ }^{1 凶 \underline{O R C I D}}$
}

Fecha correspondencia:

Recibido: enero 20 de 2020.

Aceptado: marzo 16 de 2020.

Forma de citar:

García J. Ultrasonido y accesos venosos periféricos difíciles: de las barreras al éxito, un reporte de casos. Revista CES Enfermería, 2021; 2(1): 4-20.

Open access

Creative commons

Ethics of publications

Peer review

Open Journal System

DOl: http://dx.doi.org/10.21615/

cesenferm.2.1.1

ISSNe 2745-049X

Filiación:

1. Enfermero Profesional UdeA. Magister en Bioética Universidad CES. Enfermero Fundación Clínica del Norte. Líder de Comité de Ética Hospitalaria y Ética en investigación. Coordinador suplente del centro de Investigación e innovación. Docente Facultad de Ciencias de la Salud de la Corporación Universitaria Remington. Grupo de Investigación SFC.

\section{Resumen}

Este es un reporte de serie de casos sobre accesos venosos difíciles guiados por técnica de ultrasonido, en el servicio de cirugía de la Fundación Clínica Norte en Bello, Colombia. El objetivo principal fue describir la efectividad, seguridad de la técnica ecográfica y las características clínicas de los pacientes con difícil acceso venoso. El muestreo fue consecutivo. En el muestreo final se incluyeron 14 pacientes para realizar el procedimiento ecoguiado; previamente se excluyeron 8 posibles participantes debido a venas profundas y falta de líneas medias; la principal patología en los pacientes fue la obesidad (50\%) y en segundo lugar la diabetes (28\%); en los pacientes sanos el principal factor de riesgo fue cirugías de extremidades superiores. Adicionalmente, se revisaron historias clínicas de pacientes multipuncionados, durante el tiempo estudiado (específicamente los días en que se realizaron procedimientos) encontrándose un total de 13 casos, en los cuales, se realizaron dos punciones más para canalizar un acceso venoso y no se recurrió a la valoración ecográfica. La efectividad al primer intento estuvo alrededor del $80 \%$ y las principales barreras fueron barreras humanas y técnicas, no se presentaron complicaciones asociadas. En conclusión, la ecografía es una necesidad para la canalización de accesos venosos difíciles y las enfermeras deben estar preparadas para una técnica desafiante que permite brindar atención con calidad y seguridad, conocer la técnica y familiarizarse con ella son los pasos necesarios para que integrar el ultrasonido al cuidado de enfermería.

Palabras clave: ultrasonido, dispositivos de acceso vascular, enfermería perioperatoria, ultrasonografía intravascular.

\section{Abstract}

This is a serial case report about difficult vein accesses guided by ultrasound technique in surgery service of the "CLINICA NORTE" foundation in Bello, Colombia. The main goal was to describe the effectiveness, safety of ultrasound technique and the clinical characteristics of patients with difficult venous access. The sampling was consecutive. 14 patients were included in the final sampling to perform the ultrasound-guided procedure. The main pathology in the patients was obesity (50\%), followed by diabetes (28\%). In healthy patients, the main risk factor was upper extremity surgery. Previously, 8 possible participants were excluded due to deep veins and lack of midlines. Additionally, medical records of multi- puntured patients 
were checked during the period of this study (specifically the days when ultrasound procedures were made). The main findings were, in 13 cases, more than 2 punctures were performed to cannulate a venous access and ultrasound assessment was not used. The effectiveness of the procedure guided by ultrasound was around $80 \%$ and the main barriers were human and technical. In conclusion, ultrasound is a necessity and nurses must be prepared for a challenging technique to provide care with quality and safety. Knowing and getting acquainted with ultrasound techniques are the first steps to involve ultrasound in nursing care.

Keywords: ultrasound, vascular access devices, ultrasonography, perioperative nursing.

\section{Introducción}

La frase más peligrosa del lenguaje es "Siempre lo hemos hecho asi" Grace Hopper.

La canalización de un acceso venoso periférico es el procedimiento más común en hospitales, alrededor del $70-80 \%$ de pacientes hospitalizados requiere un catéter venoso periférico, y todos los pacientes que son sometidos a procedimientos quirúrgicos con alguna técnica anestésica, requieren de un acceso vascular para la administración de profilaxis antibiótica, líquidos endovenosos, anestésicos y analgésicos intravenosos. 0 incluso, como medida de seguridad ante cualquier complicación derivada del procedimiento (1-3).

Sin embargo, obtener acceso IV puede ser difícil, incluso para personal de enfermería altamente calificado y con experiencia. Jacobson (4) tras estudiar 339 venopunciones, describió que alrededor del $15 \%$ de los pacientes requiere de una segunda punción, y en algunos pacientes no es posible realizarlo bajo las técnicas convencionales. Lo que representa un incremento en el número de punciones, mayor dolor para el paciente, aumento en la incidencia de hematomas, requerimiento de otras técnicas (catéteres centrales, valoración por radiología, entre otros) de más alto riesgo y costo, retrasos en procedimientos e incluso cancelación de cirugías; lo cual, afecta no solo al paciente y al equipo de salud, sino también a sus familias, la institución, e incluso a otros pacientes.

Existen algunos factores que dificultan la canalización periférica en los pacientes, como las pluripatologías, la obesidad, los múltiples tatuajes, el shock hipovolémico, múltiples intentos de canalización fallidos, y la necesidad de un catéter de gran calibre. Además, la confluencia de varios de estos factores hace aún más difícil el acceso vascular. Hoy en día, para identificar de una manera objetiva los pacientes con accesos vasculares difíciles existen herramientas como la DiVa E-score(1) que permite evaluar ab initio el paciente antes de una punción venosa y de acuerdo a la puntuación, ofrecer de antemano una técnica avanzada para la obtención de accesos vasculares. Es en estas situaciones más complejas donde la ecografía puede ser de ayuda $(5,6)$. De hecho, múltiples estudios han reportado la eficacia de la ecografía para la obtención de accesos vasculares, frente a la técnica convencional de palpación y observación $(6-9)$.

No obstante, históricamente la ecografía ha sido utilizada por el personal médico como técnica de diagnóstico por imagen. En el ámbito quirúrgico, el ecógrafo es una herramienta eminentemente médica, usada por anestesiólogos y cirujanos; lo 
cual, en ocasiones representa una barrera para el uso de esta herramienta por el personal de enfermería. Sin embargo, los pacientes con accesos vasculares difíciles suponen un reto, no solo para el personal de enfermería, sino también para cirujanos y anestesiólogos, pues la dificultad ante un acceso venoso difícil retrasa procedimientos y requiere de otro tipo de accesos y técnicas, en ocasiones de mayor riesgo y costo (9-11).

Se espera que este reporte de casos permita mejorar el proceso de atención de enfermería en los pacientes con acceso venoso difícil, de modo que la técnica guiada por ultrasonido sea tenida en cuenta antes de considerar accesos venosos centrales periféricos y PICC y tener el servicio de cirugía y su personal, como referente para la canalización de accesos periféricos ecoguiados.

\section{Objetivo general}

Presentar los resultados de la efectividad y seguridad del uso del ecógrafo en un grupo de pacientes con accesos venoso difícil en el servicio de cirugía de la Fundación Clínica del Norte en el periodo julio-septiembre 2020. Describiendo las características clínicas de los pacientes con accesos venosos difíciles, las complicaciones derivadas del procedimiento y las barreras para el uso del ecógrafo por parte de enfermería en pacientes con acceso venoso difícil.

\section{Metodología}

\section{Tipo de estudio}

Se realizó un estudio de reporte de serie de casos, del tipo observacional, descriptivo, en pacientes con acceso venoso difícil en el servicio de cirugía, entre el 30 de julio y el 30 de septiembre de 2020. El muestreo fue consecutivo, de modo que fuera posible incluir a todos los participantes durante el periodo estudiado, adicionalmente se dependía de la disponibilidad del personal y del equipo de ultrasonido para llevar a cabo el procedimiento. Al finalizar el estudio, se realiza una revisión de historias clínicas para valorar posibles participantes que no se incluyeron; cruzando esta información con las fechas en las que se tuvo disponibilidad de recursos humanos y de equipos.

Este estudio estuvo supeditado a la formación de los profesionales en el manejo de la técnica ecoguiada, por esta razón la alternativa de la técnica ecoguiada fue ofrecida según la disponibilidad del recurso humano y solo fue practicada por enfermeros profesionales del servicio de cirugía.

\section{Criterios de inclusión}

- Pacientes mayores de 18 años, referenciados por otro personal sanitario (médico o enfermero) como difícil acceso venoso y que se encuentren en el servicio de cirugía o en otro servicio a la espera de cirugía.

- Pacientes que se encuentren sin efectos de anestesia y puedan manifestar su participación voluntaria en el estudio y que al momento de realizar la venopunción hubiese disponibilidad del ecógrafo.

- Para el análisis retrospectivo de los posibles participantes, se incluyeron aquellos pacientes que fueron puncionados más de dos veces para la obtención de un acceso venoso y que coincidiera con disponibilidad de recurso humano y de ecografo. 


\section{Criterios de exclusión}

- Pacientes con trastornos psiquiátricos, pacientes con enfermedades vasculares o trastornos de la coagulación,

- Pacientes que tuvieron indicación de acceso vascular central o catéter venoso central de inserción periférica por su estado clínico o por el tipo y tiempo esperado del tratamiento.

- Pacientes bajo efecto de sedantes o anestésicos.

A todos los pacientes referenciados por otros profesionales o auxiliares de enfermería se les aplicó la escala EA-DiVa, la cual, es una escala práctica, rápida y útil, validada por Cívetta (1) para detectar accesos periféricos difíciles en pacientes prequirúrgicos; con un valor predictivo positivo de un $56 \%$ y un valor predictivo negativo del $97 \%$. La valoración de esta escala fue posterior a la referenciación de los pacientes, no fue el criterio de inclusión en ninguno de los casos.

Tabla 1. EA-DIVA SCORE.

\begin{tabular}{|c|c|}
\hline Característica evaluada & Puntaje \\
\hline Historia de accesos venoso difícil & Presente $=3$ Ausente $=0$ \\
\hline $\begin{array}{l}\text { Depleción de accesos vasculares debido a agentes } \\
\text { quimioterapéuticos, drogas de abuso o venopunciones previas }\end{array}$ & Presente $=2$ Ausente $=0$ \\
\hline $\begin{array}{l}\text { Desordenes de coagulación o toma de anticoagulantes o } \\
\text { antiagregantes plaquetarios }\end{array}$ & Presente $=1$ Ausente $=0$ \\
\hline Enfermedad neurovascular periférica o vasculopatía & Presente $=1$ Ausente $=0$ \\
\hline Valoración clínica de la piel, oscura, gruesa o frágil & Presente $=1$ Ausente $=0$ \\
\hline Sobrepeso (BMI>25) & Presente $=1$ Ausente $=0$ \\
\hline Venas no visibles, no palpables, curvas, vena sinuosa & Presente $=2$ Ausente $=0$ \\
\hline Disponibilidad de un solo lado o dos & Uno solo= 1 Dos $=0$ \\
\hline
\end{tabular}

En todos los participantes se utilizó el ecógrafo marca Terason, modelo Usmart 3300 con la sonda ecográfica lineal, preset mode "vascular access", con una profundidad estándar de $0-3 \mathrm{~cm}$, Se utilizaron las técnicas transversas y longitudinal para el barrido y localización inicial en todos los pacientes, se seleccionó la de mejor visualización de acuerdo con el sitio anatómico, menor profundidad y mayor relación diámetro vaso/catéter.

En algunos casos, se realizó Doppler color para diferenciar venas y arterias, todos los procedimientos fueron realizados por un operador a dos manos (enfermero profesional de cirugía). Al finalizar el procedimiento se verificó con ultrasonido localización del catéter y paso de solución salina endoluminal.

Para garantizar la esterilidad del procedimiento se utilizó apósito transparente estéril (marca Tegaderm) para proteger sonda ecográfica y evitar contaminación del sitio de punción. Adicionalmente, como reemplazo del gel transductor, se utilizó clorhexidina jabón al 2\%. 
Se tenía disponibilidad de catéteres intravenosos periféricos $22 \mathrm{~g}, 20 \mathrm{~g}, 18 \mathrm{~g}$, y $16 \mathrm{~g}$, los cuales se seleccionaron de acuerdo con el tipo de cirugía, estancia esperada, medicación a administrar, tamaño y profundidad del vaso. Se utilizaron adaptadores de terapia intermitente de presión neutra y fijación con película adhesiva estéril transparente, según protocolo institucional.

Se realizaron seguimientos a las historias clínicas de los pacientes en el postoperatorio para determinar complicaciones, duración del acceso y motivo de retiro. El seguimiento de la historia clínica se realizó a través de las notas de enfermería y control de dispositivos intravasculares, en las cuales, se describe ubicación y estado del catéter, tiempo de inserción y causa de retiro.

Durante el procedimiento ecoguiado a todos los participantes se les valoró:

- Profundidad ecográfica de la vena: resultado de la medición ecográfica en el eje transversal, de la distancia en milímetros desde la piel hasta el centro del vaso.

- Diámetro anteroposterior de la vena: resultado en milímetros de la medición ecográfica, en el eje transversal, de la distancia entre la pared anterior y posterior de la vena.

- Número de punciones previas: número de punciones realizadas al paciente antes de la valoración ecográfica con miras a la obtención de un acceso venoso periférico.

- Numero de punciones guiadas por ultrasonido: número de punciones guiadas con ultrasonido hasta la obtención de un acceso venoso periférico.

- Relación diámetro del vaso/cateter: resultado de la relación entre el diámetro del vaso (sin torniquete) y el catéter en posición endoluminal, visualizado ecográficamente en el eje transversal.

- Duración del catéter en días: número de días contados desde la inserción del cateter hasta su retiro. Para ello se realizó seguimiento posterior en las historias clínicas de los pacientes, para constatar fecha de retiro y posibles complicaciones tardías.

- Tiempo del procedimiento: Tiempo transcurrido en minutos desde el inicio del procedimiento (valoración ecográfica) hasta la fijación del catéter.

- $\quad$ EA- DiVa score: puntaje obtenido luego de la aplicación EA-DiVa score.

Adicionalmente se valoraron las variables cualitativas: tipo de cirugía por especialidad, lugar del cuerpo intervenido, tipo de procedimiento (electivo o urgente), técnica anestésica implementada, localización de la vena puncionada y comorbilidades.

Las barreras del procedimiento se clasificaron por: tiempo, equipo y personal. De acuerdo con lo planteado por Civetta (1), el tiempo asociado al procedimiento puede ser uno de los factores determinantes para usar el ecógrafo en el ámbito quirúrgico, especialmente en los procedimientos urgentes o en situaciones con una programación quirúrgica copada en la que la obtención de un acceso ecoguiado pueda retrasar 
la realización de otros procedimientos quirúrgicos, de acuerdo con los tiempos relacionados (1-3) en otros estudios, el tiempo suficiente se define como 15 minutos entre iniciar el procedimiento y el comienzo de la cirugía, o en su defecto la posibilidad de esperar a que el procedimiento se lleve a cabo. Para ello, se revisaron aquellos pacientes que pudiendo ser candidatos no se puncionaron por falta de tiempo quirúrgico o tiempo para realizar el procedimiento y que determinaron la no inclusión de participantes que cumplían con los criterios. En cuanto al equipo como barrera, se estudiaron los posibles casos de pacientes con difícil acceso venoso y que no se puncionaron por falta de equipo de ultrasonido. Es decir, aquellos casos en los que se encontraba ocupado el ecografo y adicionalmente no era esperar, o aquellos casos en los que el equipo no se encontrara en el servicio (Figura 1).

Figura 1. Diagrama de flujo disponibilidad de equipo y tiempo para realizar el procedimiento ecográfico.

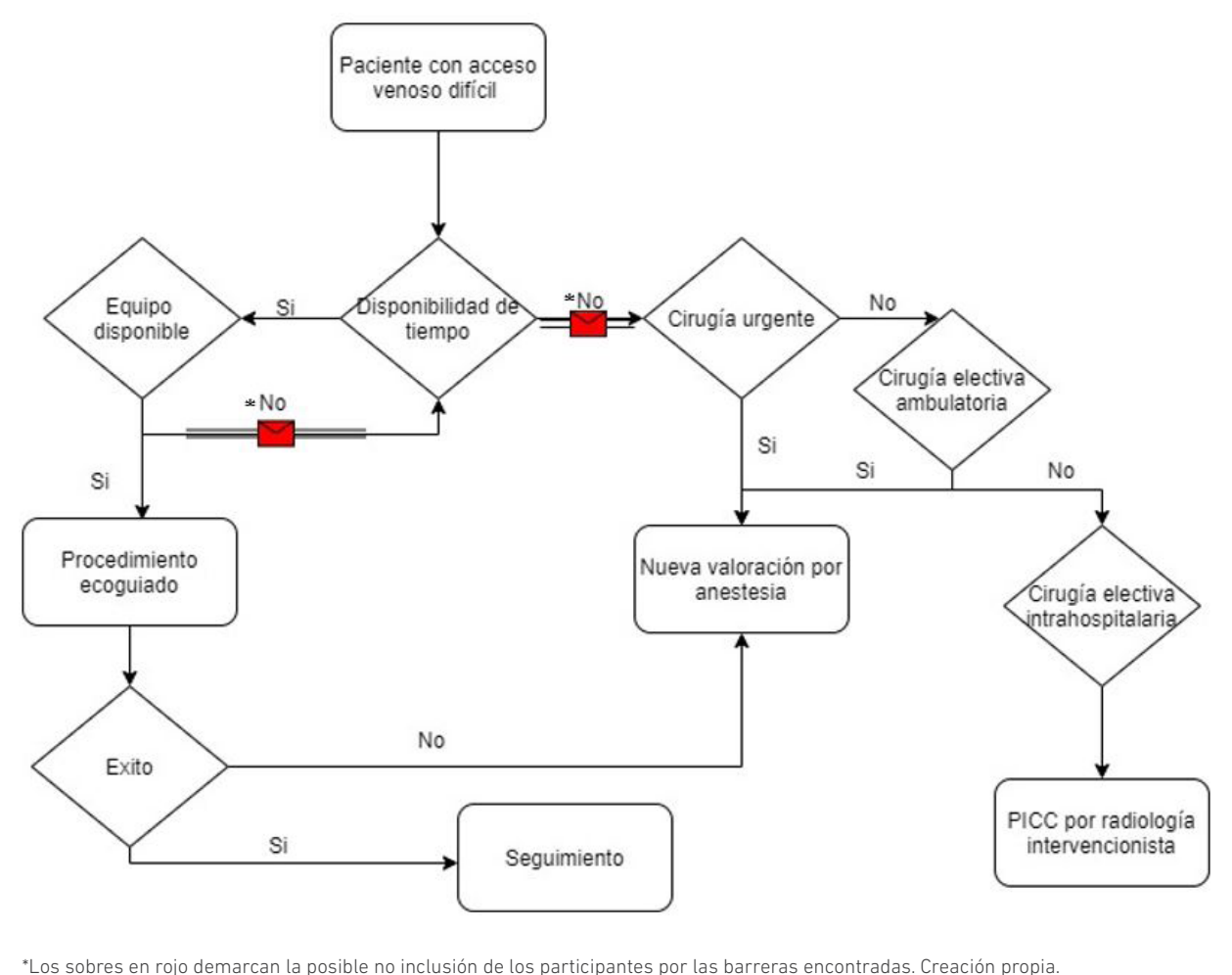

Finalmente se realiza una revisión de historias clínicas de los procedimientos quirúrgicos durante el periodo estudiado y que coincidían exactamente con los días en que se realizaron procedimientos ecoguiados. Con el fin de identificar, aquellos casos de pacientes en los que se registraron más de 2 punciones, o más de $2 \mathrm{ca}$ téteres periféricos para obtener un acceso venoso y que no se recurrió a la técnica por ultrasonido. Para ello, se filtraron las historias clínicas de los procedimientos quirúrgicos realizados bajo anestesia. Un filtro posterior, fue realizado en la sección de insumos de las historias clínicas, para dar cuenta del uso de más de 2 catéteres, y en ellas a su vez, se revisaron las notas de venopunción, para confirmar que el uso de los catéteres fuera específicamente en venopunción (también pueden usarse para irrigación quirúrgica). 


\section{Análisis estadístico}

Los datos fueron analizados en el programa estadístico SPSS versión 19, licencia propia. Las variables cualitativas se expresaron como frecuencias absolutas y relativas. Dado que la muestra fue inferior a 50, se realizó la prueba de Shapiro Wilk para hallar la normalidad del conjunto de datos, las variables normales se describen con la media y la desviación estándar, y las no normales con la mediana (me) y el rango intercuartílico (RIQ).

\section{Consideraciones éticas}

A todos los pacientes que cumplieron con los criterios y la indicación terapéutica, se les informó los objetivos de la investigación y el propósito de los resultados del estudio, dando la oportunidad y el espacio de preguntar o aclarar dudas. Una vez aclaradas las dudas; se les presentó el formato de consentimiento informado en el que aceptaron su participación en la investigación plasmando su firma o huella en este. Se garantizó la confidencialidad de la información proporcionada por los pacientes. En las historias clínicas, solo se revisaron los ítems observaciones de enfermería, informe quirúrgico y control de dispositivos intravasculares garantizando la confidencialidad de los datos obtenidos tanto de las historias clínicas como de los pacientes.

A lo largo del proceso se respetaron las normas de buena práctica clínica, se acataron las recomendaciones del Comité de Ética en investigación de la Fundación Clínica del Norte, el cual aprobó en el acta número 72, del 24 de octubre del año 2019, Este estudio fue clasificado como de riesgo mínimo.

En este sentido, todos los pacientes se trataron como fines y nunca como medios, teniendo en cuenta los principios de beneficencia, no maleficencia, justicia y autonomía. Aquellos pacientes que rechazaron su participación en el estudio, pero que se beneficiaban de la técnica guiada por ultrasonido se les realizó el procedimiento ecoguiado. Sin embargo, sus datos y hallazgos no fueron tenidos en cuenta en el estudio. De este modo, la participación en el estudio no condicionó el uso de la técnica guiada por ultrasonido.

De igual forma, ante la posibilidad de no disponer del ecógrafo siempre prevaleció el bien o el mejor interés del paciente, y si en aquel momento, el mayor beneficio lo representó otra técnica como catéter central o PICC, los pacientes fueron remitidos para la realización de dicha técnica.

\section{Resultados}

\section{Institución}

El estudio se llevó a cabo en el area de cirugía de la Fundación Clínica del Norte, Institución prestadora de Servicios de Salud (IPS) de tercer nivel de complejidad, con énfasis en cardio y neuro intervencionismo, así como en la atención del paciente politraumatizado. Las especialidades quirúrgicas de la institución son: neurocirugía, urología, ortopedia, cirugía general, cirugía cardiovascular, cirugía maxilofacial, medicina del dolor, cirugía plástica y ginecología. Se dispone de 4 salas de cirugía, una sala de procedimientos y las áreas de recuperación y preparación. El equipo de ultrasonido se encuentra en el area de preparación, pero se tuvo disponibilidad de trasladar el equipo a cualquiera de las otras áreas en caso de requerirse. 


\section{Participantes del procedimiento ecoguiado}

En el periodo evaluado se incluyeron un total 22 pacientes, de ellos 18 mujeres y 4 hombres. Después de un rastreo ecográfico inicial se excluyeron 6 pacientes por no disponibilidad de accesos puncionables (profundidad mayor a 1,2cm o diámetro $<4 \mathrm{~mm}$ ), y se excluyeron 2 pacientes por no disponibilidad del equipo; quedando un total de 14 pacientes incluidos en la muestra.

La edad de los participantes osciló en un rango entre los 23 y 89 años, con edad media de 53 años. El promedio Score EA DiVA del grupo de pacientes fue 7, lo cual indica que la puntuación estuvo cercana al punto de corte que sugiere el uso de técnicas avanzadas para accesos vasculares. Sin embargo, 4 pacientes obtuvieron un puntaje de 4 puntos; asociado a historia de acceso vascular difícil y cirugía de miembro superior, el resto de los pacientes presentó alguna patología asociada. La principal preexistencia fue la obesidad en el 50\%, la diabetes estuvo en el $28 \%$ de los casos, 3 de los pacientes obesos también fueron diabéticos, otras enfermedades que se presentaron en menor proporción fueron hipertensión arterial, cáncer y artritis reumatoide. En algunos casos confluyó más de una preexistencia (Figura 2).

Figura 2. Comorbilidades de los pacientes con accesos venosos dificiles.



Nota. Mas de una enfermedad: fueron diabetes e hipertensión (1 caso) y diabetes y artritis reumatoide.

Los 6 pacientes excluidos por la profundidad del vaso tenían como características comunes obesidad, multipuncionados y con estancia hospitalaria prolongada, por lo que se remitieron a valoración por radiología intervencionista para colocación de PICC o catéter central por especialista en anestesiología.

La figura que representa el proceso desde la selección del paciente hasta el final se puede apreciar en la figura 3. 
Figura 3. Proceso de selección, punción y seguimiento.

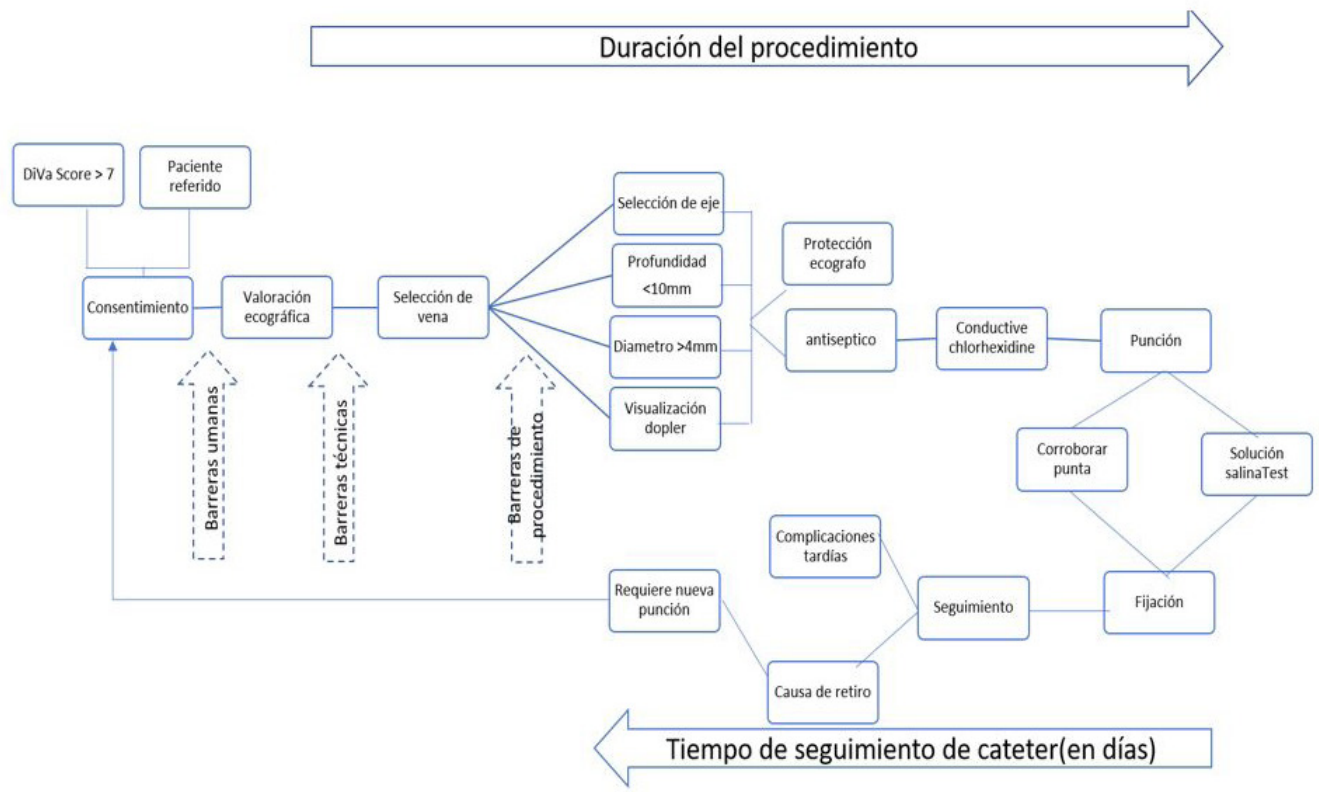

Los procedimientos quirúrgicos a los que fueron sometidos los pacientes se clasificaron por tipo de procedimiento, como electivo y urgente, los dos procedimientos clasificados como urgentes correspondieron a osteosíntesis de cuello de fémur, el resto de los procedimientos fueron electivos. También se realizó una clasificación por especialidad, tipo de cirugía y anestesia recibida (Tablas 2 y 3 ).

Tabla 2. Clasificación por especialidad y tipo de cirugía de los procedimientos quirúrgicos.

\begin{tabular}{cccc}
\hline Especialidad quirúrgica & Tipo de cirugía & N & (\%) \\
\hline Neurocirugía & Cirugías en columna* & 2 & $(14,28)$ \\
\hline \multirow{2}{*}{ Ortopedia } & Prótesis de rodilla & 3 & $(21,43)$ \\
\cline { 2 - 4 } & Osteosíntesis de fémur & 2 & $(14,28)$ \\
\cline { 2 - 4 } & Cirugías en miembros superiores* & 6 & $(42,86)$ \\
\hline Urología & Prostatectomía & 1 & $(7,14)$ \\
\hline Total & & 14 & $(100)$ \\
\hline
\end{tabular}

* Las cirugías de columna fueron artrodesis de columna torácica con fijación y lavado y desbridamiento de columna lumbar. ** Las cirugías de miembros superiores incluyeron retiro de material de osteosíntesis en humero (2), osteosíntesis de humero (3), osteosíntesis de radio y cubito (1)

Tabla 3. Tipo y subtipo de anestesia que recibieron los participantes.

\begin{tabular}{cccc}
\hline Tipo de anestesia & N & (\%) \\
\hline General & & 2 & $(14,28)$ \\
\hline \multirow{2}{*}{ Regional } & Bloqueo NP* & 6 & $(42,86)$ \\
\cline { 2 - 4 } & Raquídea & 6 & $(42,86)$ \\
\hline Total & & 14 & $(100)$ \\
\hline
\end{tabular}


Respecto a los pacientes con obesidad, 3 estaban programados para reemplazo protésico de rodilla, 2 para osteosíntesis de cuello de fémur y 2 para osteosíntesis de húmero proximal. Los pacientes de otras especialidades incluidos en el estudio no fueron obesos. Todos los pacientes participantes se encontraban en el área de preparación quirúrgica, no hubo direccionamiento de pacientes con accesos venosos difíciles de las áreas de quirófano y recuperación, por esta razón no se realizaron venopunciones ecoguiadas durante el transoperatorio ni en el postoperatorio, lo cual, se analiza en las barreras encontradas.

Los pacientes programados para cirugía urgente (2) y cirugía de columna (2) ya tenían un acceso venoso permeable, pero requerían un segundo acceso venoso; por el tipo de procedimiento y la profilaxis quirúrgica con vancomicina (para la administración de este medicamento se requiere vena exclusiva).

Adicionalmente, dos participantes se puncionaron dos veces debido a extravasación, ambos tuvieron como características comunes obesidad y vena puncionada (basílica) con una profundidad mayor a $0,9 \mathrm{~mm}$. En la segunda punción de estos pacientes, a uno de ellos se puncionó la vena yugular externa y al otro la vena cefálica, ambas con éxito.

\section{Procedimiento ecoguiado}

Se realizaron 16 venopunciones en 14 pacientes, 2 fallidas. La tasa de éxito al primer intento fue $78 \%$, y al segundo $100 \%$, en todos los pacientes que se incluyeron se obtuvo éxito, el número máximo de punciones por pacientes fue 2. El promedio de la relación vaso/catéter fue 0.135 , lo cual cumple con una relación vaso/catéter menor al 40\% evitando la oclusión total del lumen, e indica que catéteres de mayor calibre pudieron haber sido usados. El catéter número 18 fue escogido en todos los primeros intentos; pero en los intentos fallidos se optó por el catéter 20G, la profundidad media de los vasos puncionados fue $6,5 \mathrm{~mm}$, la principal complicación fue extravasación en 2 casos. El resto de las variables observadas se resume en la tabla 4.

Tabla 4. Variables observadas en los participantes con venopunción ecoguiada.

\begin{tabular}{llc}
\hline Variable & Media & (DS) \\
\hline DiVA score & 7,07 & $(3,65)$ \\
\hline Número de punciones previas & 2,36 & $(2,24)$ \\
\hline Profundidad del vaso $(\mathrm{mm})$ & 6,64 & $(2,02)$ \\
\hline Diámetro del vaso $(\mathrm{mm})$ & 8,93 & $(2,20)$ \\
\hline Duración del catéter (días) & 3,21 & $(5,15)$ \\
\hline & & \\
\hline Tiempo del procedimiento (min) & $\mathbf{M e}$ & $\mathbf{( R I Q )}$ \\
\hline Número de intentos realizados. & 11,5 & $(9)$ \\
\hline
\end{tabular}

En cuanto a la localización de la vena, no se puncionaron venas en extremidades inferiores, dado que no está permitido por políticas institucionales, excepto, en emergencia vital. Las venas del dorso de la mano tampoco se puncionaron, debido a la dificultad técnica de visualización ecográfica y una prohibición institucional relativa 
para puncionarlas; por la prevalencia de flebitis mecánica en dicha localización. La vena basílica del brazo también fue valorada ecográficamente. Sin embargo, las dos veces en que fue puncionada se extravasó y se ubicaba a una distancia cercana a un centímetro de profundidad con respecto a la piel; al no contar con catéteres periféricos largos, se hace un blanco difícil de alcanzar, el resumen de la elección del lugar a puncionar esta la descrita en la tabla 5.

Tabla 5. Distribución de frecuencias de venas puncionadas.

\begin{tabular}{lcc}
\hline Localización vena & N & (\%) \\
\hline Cefálica & 5 & $(35,7)$ \\
\hline Antebrazo & 7 & $(50,0)$ \\
\hline Yugular externa & 2 & $(14,3)$ \\
\hline Total & 14 & $(100,0)$ \\
\hline
\end{tabular}

En cuanto a la técnica ecográfica, en el corte transversal para la venopunción se utilizó en el $60 \%$ de los casos, y el longitudinal en el $40 \%$, la tasa de éxito fue similar en ambos cortes. La elección de utilizar uno u otro eje radicó principalmente en la localización del vaso, la vena cefálica y las antecubitales fueron las de mayor elección $85 \%$ de los casos. El rango de tiempo del procedimiento fue de 8 a $23 \mathrm{~min}$, no se presentaron exclusiones por tiempo quirúrgico o tiempo de procedimiento. Respecto a complicaciones posterior al procedimiento no se presentaron infecciones asociadas, la principal causa de retiro de catéter fue por protocolo institucional (92\%) y la duración media en días fue 3,2.

\section{Barreras}

Equipo

En lo que se refiere a la disponibilidad del ecógrafo, en dos casos se encontraron pacientes con acceso venoso difícil, DiVa $>7$, pero el ecógrafo se en encontraba en uso, por esta razón no fueron incluidos en este estudio y fueron puncionados por técnica palpatoria con éxito luego del tercer intento. En todos los casos se contó con los insumos necesarios. En cuanto a las barreras del procedimiento la principal limitante fue la profundidad del vaso y los catéteres cortos utilizados en la institución, 6 pacientes fueron derivados a inserción de catéter venoso central, se canceló un procedimiento por no disponibilidad de PICC, hasta el momento en la ciudad no se cuentan con líneas medias, ideales para este tipo de accesos en pacientes obesos.

Tiempo

No se presentaron problemas relacionados con el tiempo. Una dificultad técnica que se encontró y prolongó el tiempo del procedimiento fue la fijación del catéter. Dado que, luego de utilizar clorhexidina jabón, como gel conductor, para la valoración con el equipo de ultrasonido y aun después de retirar el exceso con alcohol, se observó que quedaban remanentes de jabón que dificultan la adhesión de la película de fijación.

\section{Humanas}

En cuanto a las barreras humanas, los pacientes entendieron y aceptaron el procedimiento, incluso dos de ellos requirieron nueva venopunción guiada con ecógrafo, la principal barrera humana podría atribuirse al no direccionamiento de los pacientes con accesos difíciles por parte del personal auxiliar de enfermería. 
Una vez seleccionados (de forma retrospectiva) los pacientes mutlipuncionados no incluidos en este estudio; exceptuando los pacientes incluidos en la técnica ecoguiada (14 pacientes) y aquellos excluidos del procedimiento (8 pacientes), se obtuvo un total de 13 pacientes mutlpuncionados, en los cuales, se contaba con todos los recursos (humanos, técnicos y tiempo) pero, aun así, no fueron referenciados.

En la revisión de historias clínicas, se encontraron 13 pacientes que requirieron 3 o más punciones para obtener un acceso venoso, que coincidían en las fechas en las que realizaron procedimientos ecoguiados, 8 mujeres y 5 hombres, la mediana del número de punciones de este grupo fue de 3 con un RIQ de 1 . Uno de estos pacientes requirió en el postquirúrgico la implantación de un PICC. El porcentaje de pacientes con acceso venoso difícil durante el periodo estudiado estuvo cercano al 18\%, pero de estos solo se intervino a través a través de ultrasonido el 40\% (tabla 6), el 60\% restante de estos pacientes podría haberse beneficiado de la técnica, de contar con líneas medias que permiten vasos de mayor profundidad. Pero también si se realizara un redireccionamiento de los pacientes con accesos venosos difíciles, se dispusiera mayor cantidad de enfermeros profesionales entrenados en la técnica ecoguiada y mayor disponibilidad del equipo de ultrasonido.

Tabla 6. Uso del ultrasonido en paciente con acceso venoso difícil, en la institución durante el período estudiado.

\begin{tabular}{ccc}
\hline Número punciones para & \multicolumn{2}{c}{ Ultrasonido } \\
obtener un acceso venoso & Si n (\%) & No (\%) \\
\hline$>2$ & $14(40)$ & $21(60)$ \\
$\leq 2$ & 0 & 156 \\
\hline
\end{tabular}

Los pacientes con DiVa encontrados de forma retrospectiva se clasificaron por género, tipo de procedimiento quirúrgico (tabla 7) y lugar en que se realizó el procedimiento, 4 casos fueron en recuperación y el resto (9 casos) en el área de preparación, en este grupo no fue posible valorar otras variables. Estos pacientes, no fueron redireccionados por el personal de enfermería a cargo de la venopunción; para obtener el acceso con la ayuda del ultrasonido. Conocer la causa de este comportamiento, excede los alcances de este estudio.

Tabla 7. Procedimientos quirúrgicos realizados en los pacientes multipuncionados sin ultrasonido.

\begin{tabular}{lcc}
\hline Tipo de cirugía & N & (\%) \\
\hline Colecistectomía & 1 & $(7,69)$ \\
\hline Artrodesis de columna & 2 & $(15,38)$ \\
\hline Cirugías en miembros superiores* & 6 & $(46,15)$ \\
\hline Cirugías en miembros inferiores** & 4 & $(30,77)$ \\
\hline Total & 13 & $(100)$ \\
\hline & & \\
*Las cirugías de miembros superiores incluyeron retiro de material de osteosíntesis \\
en humero (2), lavado y desbridamiento en mano o antebrazo (3), osteosíntesis de \\
radio y cubito (1). \\
$* *$ tas cirugías de miembros inferiores fueron amputaciones infra condíleas (2), os- \\
teosintesis de fémur (1) y prótesis de cadera (1).
\end{tabular}




\section{Discusión}

El éxito del procedimiento fue cercano al 100\%, los pacientes que fueron excluidos por venas demasiado profundas pudieron haberse beneficiado del procedimiento guiado por ultrasonido. Sin embargo, los catéteres periféricos largos no están disponibles en tiempos de pandemia y las líneas medias aún no están disponibles en nuestro medio.

En términos de resultados, la eficacia cercana al $80 \%$ en el primer intento es ligeramente superior a la reportada (12-14) pero similar a la realizada por Stuckey (15). En la institución el promedio de intentos de acceso venoso por paciente antes del éxito es de 1.8, y en cuanto al tiempo dedicado a procedimientos difíciles el promedio es de 10 minutos, similar a lo reportado por otros autores (14). Si bien en este estudio el tiempo promedio fue considerablemente mayor, probablemente hubiera sido mayor o imposible sin la ayuda del ecógrafo. De acuerdo con Blanco (16) el tiempo del procedimiento guiado por ultrasonido disminuye una vez se establecen los equipos de terapia intravenosa avanzada; se socializan las escalas de valoración, se reconoce el trabajo del equipo y aumenta la experticia técnica. En este sentido Ordoñez (17) afirma que la tasa de éxito aumenta con la práctica; entre 0-100 venopunciones eco guiadas la tasa de acierto se encuentra entre 0-65\%, entre 101-800 alrededor del $86 \%$ y más de 800 una tasa de éxito mayor al $90 \%$. Aunque este estudio no representa una muestra representativa, la tasa de éxito fue superior a la curva de aprendizaje descrita.

En cuanto a las complicaciones relacionadas, se encontraron dos extravasaciones, en los pacientes con accesos más profundos. De acuerdo con Shokoohi (18), la profundidad y el diámetro del vaso están íntimamente ligados con la tasa de extravasación; mayor profundidad y menor diámetro aumentan el riesgo de complicaciones. En este estudio no se puncionaron venas mayores a $12 \mathrm{~mm}$. Sin embargo, las extravasaciones se presentaron en pacientes obesos y en la vena basílica con una profundidad mayor a $10 \mathrm{~mm}$. Ante estas complicaciones o dudas del éxito en la venopunción por no retorno de sangre o dolor del paciente, la verificación de la ubicación de la punta del catéter en la luz del vaso a través del ecógrafo y la prueba de solución salina son de gran ayuda, en especial, en accesos profundos en los que se hace difícil percibir la extravasación.

De acuerdo con otros estudios (12-14,18), la canalización de accesos periféricos es un procedimiento seguro; en la medida que las complicaciones mecánicas locales incluyen hematoma, trombosis venosa superficial e infiltración local (extravasación) que solo en algunos casos provoca irritación de la piel; esto es similar a lo hallado en este estudio, dado que los dos pacientes que presentaron extravasación solo refirieron dolor al momento de realizar la prueba de soluciona salina, al realizar el seguimiento en los días posteriores no se hallaron complicaciones adicionales. No hay datos que sugieran que el uso de ultrasonido altera la tasa de estas complicaciones, pero es razonable suponer que con menos intentos requeridos gracias al uso del ultrasonido podrían posiblemente disminuirse de forma análoga las complicaciones mecánicas y el dolor $(12,18)$.

De acuerdo con algunos estudios (19-21) el desconocimiento de la técnica guiada por ultrasonido y la falta de estandarización de escalas de valoración son las principales barreras para implementar un programa avanzado de accesos vasculares. En este mismo sentido, en la medida que se implementan equipos de enfermería expertos en terapia intravenosa, se aumenta el uso de técnicas avanzadas, se reconoce el trabajo del equipo, aumentando la curva de aprendizaje y la eficacia en 
el procedimiento. De esta forma, implementar un equipo dedicado para DiVA podría reducir el tiempo de espera para la obtención de un acceso venoso, mejorar la seguridad del procedimiento, la calidad del servicio y la experticia del equipo al aumentar el flujo de pacientes que se remiten y valoran con ultrasonido.

En este mismo sentido, en este estudio no fue posible intervenir en total a 21 pacientes (60\%) y en 13 casos, aun teniendo la disponibilidad del personal, el equipo y el tiempo, estos no fueron referidos al profesional con experiencia en accesos vasculares guiados por ecografía, sino que fueron puncionados hasta alcanzar éxito. Si bien, esto no ha sido reportado en la literatura revisada, los motivos del porqué no se usa el método ecoguiado podrían abrir un camino hacia futuras investigaciones.

\section{Conclusión}

La ecografía es una herramienta muy útil para el profesional de enfermería, la falta de familiarización con este equipo y el desconocimiento al respecto, no puede ser una limitación para brindar una atención de calidad. Una vez que la enfermera demuestre dominio y experiencia, las barreras humanas desaparecerán y es probable que se la considere un profesional líder en atención de enfermería avanzada en accesos vasculares, tal como lo han reportado otros estudios (22-24). En este sentido, la creación de equipos de terapia intravenosa especializados en accesos venosos difíciles puede ser una excelente alternativa, no solo para los pacientes sino también para resaltar el rol del profesional, fortaleciendo el gremio, lo que posteriormente facilitaría la creación de sociedades y comités interinstitucionales, tal como lo han hecho en Europa (25).

Otra alternativa podría ser la inserción de una mini-línea media, según Gilardi y Giaunucci et al (26), es un procedimiento mucho más seguro y rápido cuando es guiado por ultrasonido, dado que el tiempo invertido durante el procedimiento debe utilizar una guía ecográfica en tiempo real para la colocación de vías intravenosas periféricas (PIV) en pacientes con acceso venoso periférico difícil para reducir el tiempo total del procedimiento, los intentos de inserción de la aguja y las redirecciones de la aguja. Por lo que la técnica es esencialmente la misma a los accesos periféricos y PICC, constituye una excelente alternativa.

Sin embargo, se requieren estudios con una población más amplia para comparar, tasa de éxito, curva de aprendizaje, complicaciones a largo plazo, entre otros. En cuanto a las comorbilidades de los pacientes, la tasa de pacientes sin comorbilidad y con difícil acceso venoso fue mayor que la reportada por otros estudios (20-23), podría deberse a la no evaluación de variables como el uso de fármacos intravenosos vesicantes, no valorados durante esta investigación. Se requieren más estudios posteriores para validar una escala en el contexto colombiano que permita valorar y cuidar de la mejor manera posible los pacientes con accesos venosos difíciles y poder instaurar estrategias avanzadas para la obtención de accesos vasculares. Una vez validada la herramienta, será posible estandarizar su implementación y probablemente serán más los profesionales que se interesen por conocer e implementar las técnicas ecoguiadas.

A la luz de este estudio cobra especial interés analizar en con muestras más representativas el factor de riesgo "solo disponer de una extremidad para la venopunción". Dado que, en la población quirúrgica estudiada, estuvo presente en todos en los pacientes sin otros antecedentes patológicos. 
El enfermero debe empoderarse de técnicas y tecnologías siempre encaminadas a brindar cuidado de calidad. De acuerdo con Heidegger (27), tanto el cuidado como la técnica hacen parte de la naturaleza humana. Por ello, el enfermero como agente de cuidado, ontológicamente se vale de técnicas y herramientas para cuidar.

\section{Referencias}

1. Civetta G, Cortesi S, Mancardi M, De Pirro A, Vischio M, Mazzocchi M, et al. EA-DIVA score (Enhanced Adult DIVA score): A new scale to predict difficult preoperative venous cannulation in adult surgical patients. J Vasc Access. mayo de 2019;20(3):281-9.

2. Webster J, Osborne R, Rickard C. Clinically indicated replacement versus routine replacement of peripheral venous catheters. Cochrane database of systematic reviews. 2019.

3. Rickard CM, Webster J, Wallis M. Routine versus clinically indicated replacement of peripheral intravenous catheters: a randomised controlled equivalence trial. Lancet. 2012;380(9847):1066-1074.

4. Jacobson A, Winslow E. Variables influencing intravenous catheter insertion difficulty and failure: an analysis of 339 intravenous catheter insertions. Heart lung. 2005;34(5):345-59.

5. Bodenham A. Acceso vascular. Revista Médica Clínica Las Condes. 2017;28(5): 713-26.

6. Salleras-Duran L, Fuentes-Pumarola C. Cateterización periférica ecoguiada frente a la técnica tradicional. Enfermería Clínica. septiembre de 2016;26(5):298-306.

7. Maiocco G, Coole C. Use of Ultrasound Guidance for Peripheral Intravenous Placement in Difficult-to-Access Patients: Advancing Practice With Evidence. Journal of Nursing Care Quality. 2012;27(1):51-5.

8. Weiner SG, Sarff AR, Esener DE, Shroff SD, Budhram GR, Switkowski KM, et al. Single-operator Ultrasound-guided Intravenous Line Placement by Emergency Nurses Reduces the Need for Physician Intervention in Patients with Difficult-to-establish Intravenous Access. The Journal of Emergency Medicine. 2013;44(3):653-60.

9. Presley B, Isenberg JD. Ultrasound Guided Intravenous Access. En: StatPearls [Internet]. Treasure Island (FL): StatPearls Publishing; 2020 [citado 2020 octubre 1]. Disponible en: http://www.ncbi.nlm.nih.gov/books/NBK525988/

10. Gomez S, Clemente C, Lopez M, Martinez I. Acceso venoso periférico y punción arterial guiadas por ecografía en quirófano de traumatología. Hospital Universitario Virgen de la Arrixaca.

11. Molina P, Liébana B, Moreno Y, Arribas P, Rodríguez P, Díaz P, et al. Aportación de la ecografía realizada por enfermería a la exploración del acceso vascular. Enfermería Nefrológica. 2017;20(3):241-5. 
12. Millington SJ, Hendin A, Shiloh AL, Koenig S. Better With Ultrasound: Peripheral Intravenous Catheter Insertion. Chest. 2020;157(2):369-75.

13. Franco-Sadud R, Schnobrich D, Mathews BK, Candotti C, Abdel-Ghani S, Perez $M G$, et al. Recommendations on the Use of Ultrasound Guidance for Central and Peripheral Vascular Access in Adults: A Position Statement of the Society of Hospital Medicine. J Hosp Med. 2019;14:E1-22.

14. Oliver LA, Oliver JA, Ohanyan S, Park W, Benelyahoo A, Vadivelu N. Ultrasound for peripheral and arterial access. Best Pract Res Clin Anaesthesiol. 2019;33(4): 523-37.

15. Stuckey C, Curtis MP. Development of a nurse-led ultrasound-guided peripheral intravenous program. J Vasc Nurs. 2019;37(4):246-9.

16. Blanco P. Ultrasound-guided peripheral venous cannulation in critically ill patients: a practical guideline. Ultrasound J [Internet]. 2019 [citado 2020 octubre 1]. Disponible en: https://www.ncbi.nlm.nih.gov/pmc/articles/PMC6797689/.

17. Ordoñez C, Nuño F. Canalización ecoguiada de accesos vasculares periféricos por enfermería. Revisión bibliográfica [Trabajo de posgrado]. [España]: Universidad de Zaragoza; 2019.

18. Shokoohi H, Boniface K, McCarthy M, Khedir T, Sattarian M, Ding R, et al. ULtrasound-guided peripheral intravenous access program is associated with a marked reduction in central venous catheter use in noncritically ill emergency department patients. Ann Emerg Med. 2013; 61(2):198-203.

19. Galen B, Baron S, Young S, Hall A, Berger-Spivack L, Southern W. Reducing peripherally inserted central catheters and midline catheters by training nurses in ultrasound-guided peripheral intravenous catheter placement. BMJ Qual Saf. 2020;29(3):245-9.

20. Munshey F, Parra DA, McDonnell C, Matava C. Ultrasound-guided techniques for peripheral intravenous placement in children with difficult venous Access. Pediatric Anesthesia. 2020; 13:108-15. [citado 2020 octubre 1].Disponible en: https://onlinelibrary.wiley.com/doi/abs/10.1111/pan.13780

21. Amaral M, Pettengill M. Uso de ultrasonido para guiar la punción venosa periférica en niños: significado para la enfermera. Acta paul. enferm. 2010;23(4):472478. http://dx.doi.org/10.1590/S0103-21002010000400005

22. Larsen P, Elbridge D, Brinkley J, Newton D, Goff D, Hartzog T, et al. Pediatric peripheral intravenous access: does nursing e' perience and competence really make a Difference. J Infus Nurs. 2010; 33(4):226-35. [citado 2019 octubre 10]. Disponible en: https://www.ncbi.nlm.nih.gov/m/pubmed/20631584/

23. Elkhunovich M, Barreras J, Bock Pinero V, Ziv N, Vaiyani A, Mailhot T. The use of ultrasound for peripheral IV placement by vascular access team nurses at a tertiary children's hospital. The Journal of Vascular Access. 2017;18(1):57-63. doi:10.5301/jva.5000615. 
24. Galván E, Chacon Y. Efectividad del uso de dispositivos de imagen vascular infrarrojo y ultrasonido en la canalización de acceso venoso periférico comparado con la técnica tradicional para reducir el número de intentos de punción en pacientes pediátricos [Trabajo de grado]. [Perú]: Universidad Norbert Wiener; 2019.

25. Allen L. Enfermeras Vasculares y Técnicos de Ultrasonidos EuropeosRev. enferm. vasc. 2019; 2 (3): 33. [citado 2020 octubre 13]. Disponible en: https://www. revistaevascular.es/index.php/revistaenfermeriavascular/article/view/39

26. Gilardi E, Giannuzzi R, WoldeSellasie K, Piano A, Pittiruti M, Scoppettuolo G. Mini-midline in difficult intravenous access patients in emergency department: A prospective analysis. J Vasc Access. julio de 2020;21(4):449-55.

27. Heidegger M. Being and time. Harper and Row, New York. 1988. 